\title{
Teleconsulta en otorrinolaringología durante la pandemia por COVID-19, nuestra experiencia. Hospital de Clínicas. San Lorenzo, Paraguay 2020
}

\author{
Teleconsultation in otolaryngology during the pandemic by COVID-19, \\ our experience. Hospital de Clínicas. San Lorenzo, Paraguay 2020
}

Carlos Mena C. ${ }^{1}$, Sandra Toso S. ${ }^{1}$, Raúl Tornaco M. ${ }^{1}$, Francisco Pires C. ${ }^{1}$, Annette Krauskopf B. ${ }^{1}$

\section{Resumen}

Introducción: La teleconsulta puede ser de utilidad para evitar exposición de pacientes y optimizar recursos y dirigir a los que se consideran prioridad para atención presencial. Objetivo: Valorar el grado de satisfacción de la atención por teleconsulta en el Servicio de Otorrinolaringología del Hospital de Clínicas, San Lorenzo, durante julio de 2020. Material y Método: Diseño observacional, descriptivo, corte transversal, retrospectivo, muestreo probabilístico aleatorio simple. Se realizó encuestas sobre la calidad de atención por teleconsulta. Resultados: Se contactó 86 pacientes, 78 participaron. Edad promedio 32,2 años, $58,97 \%$ mujeres. $73 \%$ era de zona urbana, $27 \%$ rural. Los motivos de consulta más frecuentes fueron obstrucción nasal, hipoacusia y molestia en garganta. Un 66,67\% no requirió ayuda para la consulta. 74,35\% evitó asistir al médico durante la pandemia, el $51,72 \%$ por prevención o miedo. El 51,28\% refirió empeoramiento de su dolencia durante la cuarentena. Se registró un alto nivel de satisfacción de la atención $(9,41 \pm 1,3)$. Un $82 \%$ refirió que esta modalidad puede ser implementada como opción, aunque el 70,5\% requirió consulta presencial. Conclusión: La mayoría de los pacientes se encontraba en rango etario económicamente activo. La mayor parte de los pacientes no necesitó ayuda para acceder a la teleconsulta, reflejando la facilidad de acceso a esta. La teleconsulta disminuyó la necesidad de consulta presencial, el grado de aceptación del sistema de teleconsulta es elevada, traducidos en los altos índices de satisfacción y aceptación de la modalidad. Palabras clave: COVID-19, teleotorrinolaringología, telemedicina, satisfacción, pandemia.

\footnotetext{
Abstract

Introduction: Teleconsultation can be useful as a triage to prevent patient exposure to the COVID-19 virus, classifying infectious ones and perfecting hospital resources, to direct those considered priority for face-to-face care. Aim: To assess the degree of satisfaction of teleconsultation care. Material and Method: Observational design study, descriptive, cross-sectional, retrospective, simple random probabilistic sampling. Surveys were conducted on the quality of care of the teleconsultation service during July 2020. Results: 86 patients were contacted, 78 were surveyed. The average age was 32.2 years, $58.97 \%$ was female. $73 \%$ came from Asunción and metropolitan area, 27\% of rural areas. Most common reasons for consultation were nasal obstruction, hearing loss and throat discomfort. $66.67 \%$ did not require help in carrying out the consultation. $74.35 \%$ avoided attending the doctor during the pandemic. $51.72 \%$ (30) for prevention or fear of infection. $51.28 \%$ reported worsening of their ailment during quarantine. There was an important level of attention satisfaction (9.41 \pm 1.3$) .82 \%$ reported that this mode of care can be implemented as an option in the service, although $70.5 \%$ require a face-to-face consultation. Conclusion: Most of the patients were in the economically active age range. Most of the patients did not required help for teleconsultation reflecting the ease of access to it. Teleconsultation reduced the need for face-to-face consultation, the degree of acceptance of the teleconsultation system is high, translated into the high rates of satisfaction and acceptance of the modality.
}

Keywords: COVID-19, tele-otolaryngology, telemedicine, satisfaction, pandemic.
${ }^{1}$ Cátedra y Servicio de Otorrinolaringología del Hospital de Clínicas. San Lorenzo, Paraguay.

Los autores declaran no tener conflictos de interés.

Recibido el 10 de septiembre de 2020. Aceptado el 31 de octubre de 2020.

Correspondencia:

Carlos Mena C.

Universidad Nacional de Asunción, Facultad de Ciencias Médicas. Mariscal López casi Cruzada de la Amistad. San Lorenzo, Paraguay. Email: cmena@med.una.py 


\section{Introducción}

Con la situación actual debido a la pandemia por el virus SARS-CoV-2, las medidas sanitarias implementadas por el Ministerio de Salud en Paraguay implicaron un cambio drástico en el modo de vivir, habiéndose declarado aislamiento preventivo general (cuarentena) el 10 de marzo de 2020, a fin de evitar la propagación del virus; el 20 de marzo se declara cuarentena total, instándose a las personas a quedarse en sus casas y evitar asistir a hospitales para evitar aglomeración de personas.

La Cátedra y Servicio de Otorrinolaringología del Hospital de Clínicas San Lorenzo siguió con las atenciones de urgencias, redactó su protocolo de manejo ante la pandemia para minimizar el riesgo de contagio y proteger al paciente siguiendo las guías ministeriales e internacionales. Debido a la creciente demanda de pacientes que requerían seguimiento por patologías crónicas y la necesidad del hospital, el cual tiene función docente en la formación de residentes, mediante sus respectivos servicios se desarrollaron estrategias para facilitar la atención a los pacientes siguiendo las normativas vigentes.

La telemedicina es una herramienta que permite al médico un contacto con el paciente prestándole una asistencia, pero a su vez previniendo, alertando y supervisando su dolencia, además, de contribuir con la vigilancia sanitaria en la contención de la propagación del virus $^{1}$. La telemedicina puede desglosarse en las modalidades de teleconsulta (facilita el acceso a la atención sin necesidad de exposición ni contacto, permitiendo al paciente ser orientado y filtrar según la necesidad de atención presencial), trabajo cooperativo o teleformación (webinars y conferencias para intercambio de información y formación continua de los profesionales de salud), telepresencia (por ejemplo, diagnósticos vía videollamada), telemonitorización (consulta para evaluar la evolución del paciente), telecirugía, entre otras $^{2}$. La teleconsulta puede ser de utilidad como un triage para evitar exposición de pacientes al virus del COVID-19, clasificando a los probables infecciosos según sus síntomas y contactos; $y$ optimizar recursos hospitalarios como equipo de protección personal y tiempo, para dirigirlos a los pacientes que se consideran prioridad para atención presencial ${ }^{3}$.

\section{Objetivo}

Valorar el grado de satisfacción de la atención por teleconsulta en el Servicio de Otorrinolaringología del Hospital de Clínicas, San Lorenzo, durante julio de 2020.

Los objetivos específicos incluyen: conocer los datos sociodemográficos de los pacientes, definir los motivos de consulta más frecuentes, valorar la accesibilidad de los pacientes al sistema de teleconsulta, determinar el grado de resolutividad del servicio de teleconsulta y determinar el porcentaje de aceptación de los pacientes con respecto a la modalidad de atención.

\section{Material y Método}

Estudio de diseño observacional, descriptivo, de corte transversal, retrospectivo. Método de muestreo probabilístico aleatorio simple. La población enfocada fue de pacientes que utilizaron el servicio de teleconsulta de la Cátedra y Servicio de Otorrinolaringología del Hospital de Clínicas. Los criterios de inclusión tomados en cuenta fueron pacientes que utilizaron el servicio de teleconsulta de la Cátedra y Servicio de Otorrinolaringología del Hospital de Clínicas en el mes de julio de 2020, sin distinción de sexo, raza y edad, cuyos datos quedaron consignados en el registro de teleconsultas y los de exclusión fueron pacientes que no accedieron a participar de la encuesta.

Con respecto a los asuntos estadísticos, el tamaño de muestra del estudio fue de 74 intervenciones, basados en el cálculo de tamaño muestral para una población de 495 teleconsultas registradas el primer mes, asumiendo una proporción del $6 \%^{2}$ con un intervalo de confianza del 95\%, asumiendo un error del 5\%.

Los instrumentos de trabajo utilizados fueron las encuestas de calidad de teleconsulta durante la pandemia realizadas mediante llamada a los pacientes que realizaron teleconsulta en el Servicio de Otorrinolaringología, durante el mes de julio de 2020 (22 días hábiles, de 7 a.m. 
a 12 p.m., de lunes a viernes), sin distinción de edad, sexo ni motivo de consulta, quienes accedieron verbalmente a participar de dicha encuesta; las encuestas fueron respondidas por el mismo paciente o encargado en el caso de menores de edad, traqueostomizados o aquellos que por fuerza mayor no pudieron realizar la consulta.

La encuesta se replicó del estudio de evaluación de consulta urológica de Leibar Tamayo y cols. ${ }^{4}$, en el cual se registra el nivel de satisfacción del paciente con respecto a la teleconsulta en general, teniendo en cuenta la accesibilidad, tiempo de espera, calidad de atención, calificado por el paciente en una escala del 1 al $10 \mathrm{y}$ cargados en tabla de datos en Microsoft Excel 2016 y el sistema EPI INFO versión 7.2.2.6. Para las pruebas estadísticas se describió la frecuencia absoluta y relativa de las variables cualitativas y cuantitativas. Los datos fueron procesados y presentados en gráficos donde se utilizaron medidas de tendencia central. La aleatorización se llevó a cabo utilizando el software gratuito $O x M a R^{5}$.

Los datos que se recabaron de las encuestas no incluyeron información que permitiera identificar la identidad de los pacientes, respetando su confidencialidad. Se respetó los principios de beneficencia, es decir, en caso de que se haya percatado información faltante o resultado de alarma, los investigadores se encargaron de informar a los pacientes y/o médicos encargados del servicio; no maleficencia, los datos obtenidos en esta encuesta no se utilizaron para otros fines ajenos a la investigación en este estudio; justicia, puesto que todos los pacientes tuvieron la misma oportunidad de participar en el estudio y autonomía, contando con la autorización de la jefatura del Hospital de Clínicas para el desarrollo de este estudio.

\section{Resultados}

Se contactó a 86 pacientes, de los cuales 78 accedieron a participar de la encuesta telefónica. La edad promedio de los pacientes encuestados que requirieron el servicio de teleconsulta fue de 32,2 años con un rango de 1 año a 86 años, siendo el 58,97\% del sexo femenino. El 73\% de los pacientes del estudio provenían de Asunción y área metropolitana, el
27\% de área rural. La distribución de consultas por especialidad se muestra en la Figura 1.

En cuanto a los motivos de consulta más frecuentes se detallan en la Figura 2, en otros, se destacan disfonía, tumoración cervical, mareos, controles postquirúrgicos, entre otros. El $66,67 \%$ de los usuarios del servicio no requirió ayuda para llevar a cabo la consulta, mientras que el $33,33 \%$ no pudo llevarla a cabo por su cuenta. El 74,35\% de los pacientes evitó asistir al médico durante la pandemia. El 51,72\% por prevención o miedo al contagio; el 24,13\% por disposición ministerial de suspensión de consultas no urgentes, otras causas menos frecuentes fueron la escasez de recursos o transporte para acudir al médico, entre otras. El 48,71\% de los usuarios refirió haber perdido alguna consulta, estudio o procedimiento médico y el $51,28 \%$ de los usuarios refirió tener sensación de que su dolencia empeoró durante la cuarentena.

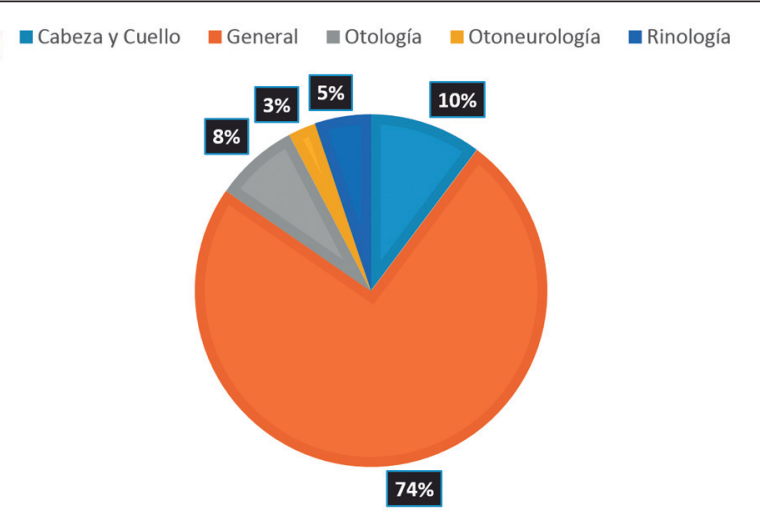

Figura 1. Distribución de consultas por especialidad $(n=78)$. Teleconsulta en otorrinolaringología durante la pandemia por COVID-19, nuestra experiencia. Hospital de Clínicas. San Lorenzo, Paraguay 2020.

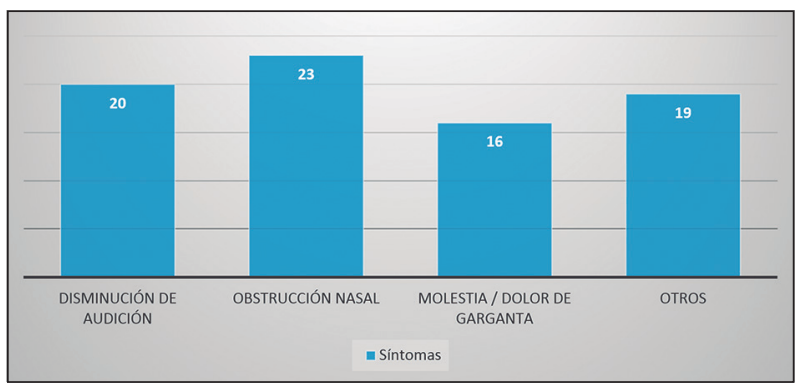

Figura 2. Motivos de consulta más frecuente $(n=78)$. Teleconsulta en otorrinolaringología durante la pandemia por COVID-19. Hospital de Clínicas, San Lorenzo, Paraguay 2020. 
En cuanto a la teleconsulta, el 89,74\% de los usuarios refirió que se empleó el tiempo adecuado para su atención. El promedio de valoración de claridad de información ofrecida durante la consulta fue de 9,14 1,12. La media de valoración del trato y amabilidad del médico durante la consulta fue de $9,5 \pm 0,8$. El 84,61\% de los encuestados refirió que el horario de atención de la teleconsulta es adecuado, y la media de valoración de la satisfacción de la atención fue de $9,41 \pm 1,3$, registrando un alto nivel de satisfacción. El 82\% de los sujetos del estudio refirió que esta modalidad de atención puede ser implementada como una opción en el servicio, aunque el 70,5\% no obtuvo solución de su dolencia por esta vía y requirió una consulta presencial.

\section{Discusión}

El contexto nacional actual obliga a los servicios sanitarios a depender de un organigrama orquestado por el Ministerio de Salud, aunque pobremente equipados y provistos de infraestructura, recursos y capacitación del personal, que deja en evidencia la poca preparación de los sistemas de salud para casos excepcionales como una pandemia ${ }^{6}$.

Los servicios de salud, para seguir ofreciendo atención al público en general, desarrollaron estrategias para la atención universal de pacientes. La Cátedra y Servicio de Otorrinolaringología del Hospital de Clínicas, mediante autogestión, fue dotado de equipos para este efecto, llevando a cabo así la atención mediante mensajería, llamadas, videollamadas vía plataforma WhatsApp y la aplicación Medicasa, puesto que estos son importantes para que el paciente pueda mostrar fotos de lesiones, resultados de estudios, etc. ${ }^{7}$.

Se registró durante el primer mes de experiencia 495 consultas por teleconsulta (22 días hábiles), cifras similares, pero ligeramente por debajo de las registradas en el estudio realizado por los Servicios de Otorrinolaringología y Cirugía de Cabeza y Cuello del Hospital Morales Meseguer de Murcia y del Hospital Virgen del Castillo, con más de mil teleconsultas entre ambos centros, en un periodo de 18 días hábiles ${ }^{8}$.

La atención por teleconsulta en nuestro ser- vicio, así como el estudio de Martínez García y cols., ayuda a redistribuir el flujo de pacientes, haciéndolo más ordenado, exceptuando las consultas por urgencias, para lo cual se seguirá planteando estrategias a fin de optimizarlas; nuestra tasa de resolución fue ligeramente mayor $(29,5 \%)$ a la del estudio español de hasta $23,98 \%$ en caso de primeras consultas 9 Nuestro estudio no valoró la relación costo-beneficio de la atención por teleconsulta versus la presencial, pero existen estudios que reportan cifras beneficiosas en favor de la teleconsulta en otorrinolaringología ${ }^{10}$.

En este estudio, los motivos de consulta más frecuentes fueron la disminución de audición y síntomas obstructivos nasales, los cuales (hipoacusia, seguido de dificultad respiratoria nasal) también fueron los más frecuentes en el estudio de Tenor Serrano y cols. ${ }^{11}$.

Otorrinolaringología es una especialidad que explora principalmente cavidades (nasal, conducto auditivo, fauces), por lo que es de esperarse que requiera un examen físico más minucioso, no completamente accesible por esta modalidad de consulta, pero ello no le resta importancia a la capacidad de la teleconsulta de determinar qué pacientes requieren una atención presencial y cuáles de manera más o menos diferida, permitiéndonos seguir atendiendo a pacientes previamente seleccionados buscando protegerlos así como al personal, minimizando la exposición ${ }^{12}$.

La aceptación de la teleconsulta como opción de atención fue del $82 \%$ en este estudio, así como un alto grado de satisfacción, en contraste con el estudio de Eaton y cols., que vieron menor tasa de satisfacción, aunque en una población diferente a la nuestra y en un contexto distinto (previo a la pandemia por COVID-19), lo cual puede constituir un sesgo debido al miedo de la gente a acudir a los hospitales como un atractivo a favor de la teleconsulta ${ }^{13}$.

Una dificultad de la teleconsulta en nuestro país es la falta de acceso universal a internet, especialmente en área rural, donde debería ser de mayor utilidad debido a la escasez de especialistas; aunque es una herramienta prometedora y en creciente difusión en nuestro medio y de gran aceptación por parte de los pacientes. Al ser el único servicio público en ofrecer servicio de otorrinolaringología en el 
país con urgencias las 24 horas, es colapsable ante una mayor demanda, que se dará eventualmente debido a la difusión en los medios masivos de comunicación, por lo que nos vemos obligados por el compromiso social de la institución en seguir desarrollando estrategias para ofrecer un servicio de calidad y a la mayor población posible ${ }^{14}$.

En este estudio tan solo el $27 \%$ era de áreas rurales, problema también referido por un estudio venezolano, en el que se augura que este tipo de prácticas ayuda a disminuir la brecha digital, mediante educación y motivando a las poblaciones a utilizar dichas herramientas ${ }^{15}$. Un tercio de los pacientes de este estudio no pudo llevar a cabo la consulta sin ayuda de algún familiar, en contraste con el 18\% de los pacientes del estudio de referencia en un servicio de urología ${ }^{4}$.

Una limitación del estudio es el hecho de que el staff de teleconsulta conformado por 10 médicos, algunos de ellos sin manejo del idioma Guaraní, lengua materna de gran porcentaje de la población (ya que el Paraguay es una nación que posee dos idiomas oficiales) $\mathrm{y}$, por ende, de los pacientes, podrían sesgar las valoraciones respecto a claridad de la información ${ }^{16}$.

\section{Conclusión}

El promedio de edad de los 78 usuarios que accedieron a participar de la encuesta fue de 32,2 años, la mayoría dentro del rango etario económicamente activo, siendo un porcentaje no despreciable de zonas rurales, quienes tienen cada vez más acceso a la tecnología. Entre los principales motivos de consulta se encontraban los síntomas obstructivos nasales, disminución de audición y molestia o dolor de garganta, los cuales de por sí son motivos frecuentes de consulta en la especialidad.

El 2/3 de los pacientes no tuvo inconvenientes para acceder a la teleconsulta y no requirió ayuda para el efecto, lo que refleja la facilidad de acceso a ella. Aunque menos del $30 \%$ de los pacientes no requirió una consulta presencial, siendo reflejo de la resolución del cuadro mediante la consulta a distancia, el grado de aceptación del servicio de teleconsulta es elevado en nuestros encuestados, traducidos en los altos índices de satisfacción y aceptación de la modalidad.

\section{Bibliografía}

1. Cabral MB, Galván P, Cane V. Telemedicina: metas y aplicaciones. Mem Inst Investig Cienc Salud. 2008;6(1):40-44.

2. Monteagudo JL, Serrano L, Hernández Salvador C. La telemedicina: ¿ciencia o ficción? Anales Sis San Navarra. 2005;28(3):309-323.

3. Márquez V, Ricardo J. Teleconsulta en la pandemia por Coronavirus: desafíos para la telemedicina posCOVID-19. Rev Col Gastroenterol. 2020;35(1):5-16. doi: 10.22516/25007440.543.

4. Leibar Tamayo A, Linares Espinós E, Ríos González E, Trelles Guzmán C, Álvarez-Maestro M, de Castro Guerín C, et al. Evaluación de la teleconsulta en el paciente urológico durante la pandemia COVID-19. Actas Urol Esp. 2020 (En prensa). doi: 10.1016 / j.acuro.2020.06.002.

5. Guillaumes S, O’Callaghan C. Versión en español del software gratuito OxMaR para minimización y aleatorización de estudios clínicos. Gac Sanit. 2019;33(4):395-397. doi: 10.1016/j. gaceta.2018.07.013.

6. Velázquez M, Pacheco A, Silva M, Sosa D. Evaluación del proceso de teleconsulta desde la perspectiva del proveedor, Programa de Telesalud de Oaxaca, México. Rev Panam Salud Publica. 2017;41e22. doi: 10.26633/RPSP.2017.22.

7. Rodríguez JI, Contreras E, García M, García L, Granero J. Telemedicina, telementorización y evaluación telemática en cirugía. ¿Es su momento después de la COVID-19? Cir Esp. 2020; doi: 10.1016/j.ciresp.2020.05.022.

8. Gómez M, Piqueras F, Guillamón L, Galindo L, Jara A, Martínez J. Gestión de la consulta de otorrinolaringología durante el estado de alarma por la pandemia del COVID-19. ¿Es útil la consulta telefónica en ORL? Acta Otorrinolaringológica Esp. 2020; doi: 10.1016/j.otorri.2020.06.001.

9. Martínez M, Bal M, Santos F, Ares R, Suárez $\mathrm{R}$, Rodríguez A, et al. Telemedicina con telemonitorización en el seguimiento de pacientes con COVID-19. Rev Clin Esp. 2020; doi: 10.1016/j. rce.2020.05.013.

10. Anari S, Arullendran P, Reilly J. How we do it: cost-benefit analysis of implementing a telephone review system in an ENT outpatient setting. Clin Otolaryngol. 2006;31(4):331-4. doi: 10.1111/j.17494486.2006.01193.x.

11. Tenor R, Plata C, Colomo N, Conde M, Oliva M. Motivos de consulta de pacientes atendidos en un 
servicio de ORL en un hospital de segundo nivel. Rev ORL. 2016 ;7(4): 205-10. doi:10.14201/orl.14638.

12. Pérez I, Santamaría S, Romero G, Vergara J. Telemedicina en la práctica del otorrinolaringólogo en el período de contingencia del COVID-19. Acta de Otorrinolaringología \& Cirugía de Cabeza y Cuello. 2020;48(1):37-44. doi: 10.37076/acorl.v48i1.488.

13. Eaton J, Rasgon B, Derbin L, Hilsinger R, Buenavista T. Telephone Appointment Visits for Head and Neck Surgery Follow-up Care. Laryngoscope. 2002;112(6):1060-4. doi: 10.1097/00005537200206000-00023.
14. Vera J, Otero L, Turrubiates E. Telemedicina y teleotorrinolaringología: Un proyecto factible para el Servicio de Sanidad Militar en México. Rev Sanid Milit Mex. 2010;64(2):79-83.

15. Valero G, Briceño L, Sanabria T. La telemedicina en las medicaturas rurales en Venezuela. Gac. Méd. Caracas. 2009;117(2):111-7.

16. Von Streber G. Paraguay and the Complexities of a Bilingual Nation: The Contradiction of the Guarani Language as a National Symbol and its Condition of Diglossia. Encuentros. 2018;16(1):107-19. doi: 10.15665/.v16i01.1401. 